\title{
An evidence-based, risk-adapted algorithm for anti-fungal prophylaxis reduces risk for invasive mold infections in children with hematologic malignancies
}

\author{
Ashley Ikwuezunma ${ }^{1}$, Ankhi Dutta ${ }^{2}$, Maria Castellanos ${ }^{2}$, Julienne Brackett ${ }^{2}$, Kiranmye \\ Reddy $^{2}$, Priya Mahajan ${ }^{2}$, Ann Marshburn ${ }^{3}$, Kala Kamdar ${ }^{2}$, Hana Paek ${ }^{4}$, Debra Palazzi ${ }^{2}$, \\ Karen Rabin ${ }^{2}$, Michael Scheurer ${ }^{2}$, and Maria Gramatges ${ }^{2}$ \\ ${ }^{1}$ The University of Texas Rio Grande Valley School of Medicine \\ ${ }^{2}$ Baylor College of Medicine Department of Pediatrics \\ ${ }^{3}$ University of Texas Health Science Center at Houston \\ ${ }^{4}$ Texas Children's Cancer Center and Hematology Centers
}

May 5, 2021

\begin{abstract}
Background: Children with hematologic malignancies, especially those who receive intensive chemotherapy, are at high risk for invasive mold infections (IMI) that confer substantial mortality. Randomized controlled trials support the use of anti-fungal prophylaxis with anti-mold activity as an optimal strategy for risk reduction in this population, but studies outlining the practical application of evidence-based recommendations are lacking. Procedure: We conducted a 15-year, single-institution retrospective review of children with hematologic malignancies treated with chemotherapy to determine the incidence of proven or probable IMI diagnosed between 2006 and 2020 and to identify the host and disease factors associated with IMI risk. We then compared the incidence and type of IMI and related factors before and after 2016 implementation of an evidence-based, risk-adapted anti-fungal prophylaxis algorithm that broadened coverage to include molds in patients at highest risk for IMI. Multivariable linear regression was used to determine factors related to IMI risk. Results: We identified 61 cases of proven or probable IMI in 1,456 patients diagnosed with hematologic malignancies during the study period (4.2\%). Implementation of an anti-fungal prophylaxis algorithm reduced the IMI incidence in this population from $4.8 \%$ to $2.9 \%$. After multivariable analysis, both Hispanic ethnicity and cancer diagnosis prior to 2016 were significantly associated with risk for IMI. Conclusion: An evidence-based, risk-adapted approach to anti-fungal prophylaxis for children with hematologic malignancies is an effective strategy to reduce incidence of IMI.
\end{abstract}

An evidence-based, risk-adapted algorithm for antifungal prophylaxis reduces risk for invasive mold infections in children with hematologic malignancies^

Ankhi Dutta, MD, $\mathrm{MPH}^{1 *}$ and Ashley Ikwuezunma, $\mathrm{BS}^{2 *}$; Maria I. Castellanos, $\mathrm{MD}^{3}$; Julienne Brackett, $\mathrm{MD}^{3}$; Kiranmye Reddy, $\mathrm{MD}^{3}$; Priya Mahajan, $\mathrm{MD}^{3}$; Ann M. Marshburn, $\mathrm{MD}^{4}$; Kala Kamdar, $\mathrm{MD}^{3}$; Hana Paek, PharmD ${ }^{5}$; Debra L. Palazzi, MD, $\mathrm{MEd}^{1}$; Karen R. Rabin, MD, PhD ${ }^{3}$; Michael E. Scheurer, PhD, $\mathrm{MPH}^{3}$; Maria M. Gramatges, $\mathrm{MD}, \mathrm{PhD}^{3}$

*contributed equally

${ }^{1}$ Department of Pediatrics, Section of Infectious Diseases, Texas Children's Hospital, Baylor College of Medicine

${ }^{2}$ University of Texas Medical School, Rio Grande Valley 
${ }^{3}$ Department of Pediatrics, Section of Hematology and Oncology, Texas Children's Hospital, Baylor College of Medicine

${ }^{4}$ Department of Pediatrics, Division of Pediatric Hospital Medicine, UTHealth Science Center

${ }^{5}$ Department of Pharmacy, Texas Children's Hospital

Corresponding Author:

Maria Monica Gramatges, MD, PhD

Feigin Tower, Baylor College of Medicine

1102 Bates St, Ste 1200

Houston, TX 77030

(p) 832-824-4678; (e) gramatge@bcm.edu; (f) 832-825-4651

Word Count: (abstract $228, \max 250$ ) (main text 2809 , max 3500)

Number of Tables, Figures, Supporting Information: 6 ( 5 tables and 1 figure)

Dutta at the IDWeek annual meeting in 2016 ('Epidemiology, management and outcome of invasive mold infections in pediatric hematological malignancies at a tertiary care center: a 10 year review'), and by Ms. Ikwuezunma at the American Society of Hematology annual meeting in 2020 ('Antifungal prophylaxis and risk for invasive mold infections in children with hematologic malignancies').

Running Title: Mold Infections in Pediatric Leukemia and Lymphoma

Keywords: antifungal prophylaxis, leukemia, lymphoma, invasive mold infection

Abbreviations:

ALL acute lymphoblastic leukemia

AML acute myeloid leukemia

EORTC/MSG European Organization for Research and Treatment of Cancer/Mycoses Study Group

HIM Health Information Management

HSCT hematopoietic stem cell transplantation

IFD invasive fungal disease

IMI invasive mold infection

TCH Texas Children's Hospital

\section{ABSTRACT}

Background: Children with hematologic malignancies, especially those who receive intensive chemotherapy, are at high risk for invasive mold infections (IMI) that confer substantial mortality. Randomized controlled trials support the use of antifungal prophylaxis with anti-mold activity as an optimal strategy for risk reduction in this population, but studies outlining the practical application of evidence-based recommendations are lacking.

Procedure: We conducted a 15-year, single-institution retrospective review of children with hematologic malignancies treated with chemotherapy to determine the incidence of proven or probable IMI diagnosed between 2006 and 2020 and to identify the host and disease factors associated with IMI risk. We then compared the incidence and type of IMI and related factors before and after 2016 implementation of an evidence-based, risk-adapted antifungal prophylaxis algorithm that broadened coverage to include molds in 
patients at highest risk for IMI. Multivariable linear regression was used to determine factors related to IMI risk.

Results: We identified 61 cases of proven or probable IMI in 1,456 patients diagnosed with hematologic malignancies during the study period (4.2\%). Implementation of an antifungal prophylaxis algorithm reduced the IMI incidence in this population from $4.8 \%$ to $2.9 \%$. After multivariable analysis, both Hispanic ethnicity and cancer diagnosis prior to 2016 were significantly associated with risk for IMI.

Conclusion: An evidence-based, risk-adapted approach to antifungal prophylaxis for children with hematologic malignancies is an effective strategy to reduce incidence of IMI.

\section{INTRODUCTION}

Invasive fungal disease (IFD) is a leading cause of mortality in immunocompromised hosts. In children diagnosed with cancer and treated with chemotherapy alone, risk for IFD is augmented by prolonged periods of neutropenia, exposure to specific high-intensity treatment regimens, and systemic corticosteroid use. Risk is also higher among children diagnosed with high-risk acute lymphoblastic leukemia (ALL), acute myeloid leukemia (AML), and relapsed disease. ${ }^{1,2}$

Among children with hematologic malignancies, the incidence of IFD varies from about $5-10 \%$ according to host factors, intensity of the chemotherapy regimen, and historical inconsistencies in supportive care practices. ${ }^{3}$ Randomized controlled trials conducted in children support the use of mold-active azoles over no systemic antifungal prophylaxis, and over fluconazole prophylaxis in at-risk children undergoing treatment for cancer. ${ }^{4-7}$ Based on this evidence, national clinical practice guidelines were developed for systemic antifungal prophylaxis in children undergoing treatment for cancer and recipients of hematopoietic stem cell transplant (HSCT). ${ }^{8}$ Specific recommendations for children with hematologic malignancies not treated with HSCT include administering antifungal prophylaxis to children with AML (strong) and to newly diagnosed or relapsed patients with ALL at high risk for IFD (weak) using a mold-active agent (echinocandin or moldactive azole, strong), particularly during periods of anticipated neutropenia (weak). However, the variability in local fungal organism epidemiology and variation in approaches to leukemia therapy suggest the need for site and protocol-specific adaptation of these guidelines to both facilitate clinical application and minimize risk for IFD. ${ }^{8}$

We conducted a single-institution retrospective review of children diagnosed with ALL, AML, and lymphoma who developed proven or probable IFD, with a specific focus on invasive mold infections (IMI). Our objective was to assess the IMI incidence and epidemiology pre- and post-implementation of a risk-adapted, local epidemiology-informed algorithm for determining antifungal prophylaxis in children undergoing treatment for hematologic malignancies.

\section{METHODS}

\section{Population and Study Design}

This retrospective observational study included children [?] 21 years diagnosed at Texas Children's Hospital (TCH) with ALL, AML, or lymphoma between 2006 and 2019, and who had a proven or probable IMI diagnosed between January 1, 2006 and June 30, 2020. Recipients of HSCT who developed IMI post-HSCT were excluded. The TCH antifungal algorithm was implemented in January 2016, and comparisons were made between time periods of January 2006 through December 2015, and January 2016 through June 2020. Potential cases were identified by the TCH Health Information Management (HIM) team using ICD9 codes that included ALL and AML (ICD9 204-208), lymphoma (ICD9 200-202), and fungal disease (ICD9 117-118, $421,518.89,530.19,730.2,739.19)$. The study was approved by the Baylor College of Medicine institutional review board.

\section{Data Collection}

The potential case list obtained from HIM was first reviewed to remove duplicate cases, confirm cancer diagnosis inclusion criteria (ALL, AML, or lymphoma), and exclude any patients with underlying immunod- 
eficiency or who developed IMI after HSCT or organ transplant. For the remaining patients, demographic, laboratory, clinical and radiographic data were manually abstracted from the electronic medical record system using a standardized, comprehensive data abstraction form. Demographic and clinical characteristics of the total number of cases diagnosed during the study time period were obtained from records maintained by the clinical disease-based teams and verified in the medical record.

All remaining potential IMI cases were then classified as meeting or not meeting criteria for probable or proven IMI, as defined by the 2008 European Organization for Research and Treatment of Cancer/Mycoses Study Group (EORTC/MSG) Consensus Group. ${ }^{9}$ To make this determination, records were reviewed by two independent members of the research team (AD, JB, KR, PH, AM, AI, MC), and discordant entries were resolved through group discussion. All mold isolates noted in the medical record were identified according to standard laboratory practices. The date of diagnosis of IMI was defined as the date of first positive culture and/or diagnostic study. Clinical information regarding the underlying disease status (date of cancer diagnosis, type of cancer, and whether the patient was receiving upfront treatment or treatment for relapsed disease), relevant exposures concurrent to the diagnosis of IMI, and relevant host factors were abstracted for all patients. Factors previously established as risk factors for IMI were noted if present within 2 weeks of the IMI diagnosis, such as neutropenia (at least one incidence of absolute neutrophil count $<500$ cells $/ \mathrm{mm}^{3}$ ), hyperglycemia (at least one incidence of random blood glucose level $>200 \mathrm{mg} / \mathrm{dl}$ ), intensive care unit admission, and concomitant bacterial infection(s) requiring antibiotics.

\section{Statistical analyses}

Descriptive statistics were used to summarize patient characteristics. Host and disease-related factors, as well as IMI incidence, were compared between patients treated from 2006-2015 and those treated from 20162020 using a Chi-square, Fisher's exact, or Student t-test, as appropriate. Host and disease related factors predictive of IMI for patients with and without a diagnosis of proven/probable IMI over the entire time period (2006-2020) were assessed by univariable and multivariable linear regression. For all comparisons, p values were considered significant at alpha $<0.05$. All statistical analyses were performed using Intercooled Stata, version 14 (Stata Corp, College Station, TX).

\section{RESULTS}

Over the 15 year study period, there were 1,456 patients diagnosed with hematologic malignancies at TCH: 981 with ALL, 173 with AML, and 302 with lymphoma. The HIM search provided a list of 365 potential patients that met our ICD9 code inclusion criteria for both hematologic malignancy and IMI. After medical record review, 61 patients $(4.2 \%)$ met criteria for pre-HSCT proven or probable IMI as defined by the EORTC/MSG, including 8 cases of probable IMI and 53 cases of proven IMI. The incidence of IMI varied by cancer diagnosis, with highest incidence in AML (11/173, 6.4\%), then ALL (45/981, 4.6\%), and lowest incidence in children with lymphoma $(5 / 302,1.7 \%)$. The distribution of patient characteristics who were diagnosed with leukemia or lymphoma between 2006 and 2019 is shown in Table $\mathbf{1}$, compared with the subset who were also diagnosed with IMI, showing a higher likelihood of AML and lower likelihood of lymphoma in the IMI subgroup.

\section{Adaptation of clinical guidelines to develop a risk-based algorithm for antifungal prophylaxis}

Development of an evidence-based algorithm for antifungal prophylaxis, including broader coverage prophylaxis, was initially prompted by a relatively high incidence of IMI noted in specific leukemia patient populations treated prior to 2016 compared with reported frequencies of IMI in the published literature. Prior to 2016, routine antifungal prophylaxis was employed for a limited population of children diagnosed with high-risk hematologic malignancies. For example, all children diagnosed with AML were prescribed fluconazole, but there was inconsistent practice for prescribing prophylaxis to children with relapsed disease or in other high risk groups. If antifungal prophylaxis was strongly recommended on a particular clinical trial, such prophylaxis was given to all patients treated according to that regimen, regardless of their enrollment to the research study. In 2015, we established a multidisciplinary team of TCH Cancer and Hematology Center Leukemia team providers, clinical pharmacy specialists, and members of the TCH Infectious Diseases 
team to identify and address factors that may be contributing to excess IMI in children with hematologic malignancies. The team reviewed incident cases in patients with oncologic diagnoses, and hospital-wide patterns of mycoses epidemiology. Available evidence-based guidelines were reviewed, considering specific at-risk populations and perceived intensity of chemotherapy treatment, prior to reaching a consensus for a risk-based algorithm and flow chart (Figure 1 ). This clinical resource was finalized and implemented in January of 2016, an effort that included delivery of section-wide education to TCH Cancer and Hematology Center faculty and fellows.

Change in IMI frequency pre and post-implementation of an antifungal risk-based algorithm

Implementation of an evidence-based, risk-adapted algorithm for antifungal prophylaxis reduced the frequency of IMI in patients with hematologic malignancies by $40 \%$, from $4.8 \%$ to $2.9 \%$. The decrease in IMI incidence was most pronounced among patients with AML, likely due to a change in practice that broadened antifungal coverage to include molds (Table 2 ).

Patient characteristics pre and post-implementation of an antifungal risk-based algorithm

There were no significant differences in sex, race, ethnicity, or cancer diagnosis between patients diagnosed before or after algorithm implementation, nor did we observe any change in patterns of IMI timing (in first remission vs. relapse, age at IMI diagnosis, Table 1 ). Host and clinical risk factors known to be associated with risk for IMI were also largely unchanged between the two time periods (Table 3 ), except that patients treated more recently were more likely to be admitted to the intensive care unit $(\mathrm{p}=0.003)$.

Fungal isolates pre- and post-implementation of the TCH antifungal algorithm

Fifty-three out of 61 IMI cases diagnosed from 2006-2020 had a proven IMI: 47 had a single mold isolate, and six $(11.3 \%)$ had multiple mold organisms isolated. The most common mold observed overall was Aspergillus species ( $\mathrm{n}=19 ; 35.8 \%)$. All Aspergillus IMI occurred prior to algorithm implementation, as did all of the cases with multiple organisms isolated. The absence of Aspergillus cases from 2016-2020 was further verified by reviewing the mold isolate log in the TCH Microbiology laboratory Infectious Disease database. Post-algorithm implementation, the most common molds isolated included Trichosporon $(\mathrm{n}=5)$ and Rhizopus $(\mathrm{n}=4)$ among the total of 14 molds identified from 2016 to 2020 (Table 4 ).

The impact of IMI on patient mortality

The overall survival of children with hematologic malignancy and IMI was $57 \%$ (35 out of 61 have survived to date, with a minimum of two years of follow up since cancer diagnosis). For the 11 AML cases with IMI, only 3 survived (27\%), and for the 45 ALL cases with IMI, 30 survived (67\%). Of the 61 IMIs diagnosed between 2006 and 2020, 14 occurred in patients with relapsed/refractory leukemia or lymphoma, and 47 occurred in patients who were in clinical remission. Unsurprisingly, 12/14 (86\%) relapsed/refractory patients died, vs. $14 / 47(30 \%)$ patients who were in remission at the time of their IMI diagnosis $(\mathrm{p}=0.0002)$. Eighteen out of the 26 patients who died had active fungal disease at the time of their death (69\%). There was no notable difference in mortality risk pre- and post-implementation of the TCH antifungal algorithm. Of the 47 IMI cases diagnosed from 2006-2015, $20(43 \%)$ died and $13(65 \%)$ had active fungal disease at the time of their death. Out of the 14 IMI cases diagnosed from 2016-2020: 6 (43\%) died and 5 (83\%) had active fungal disease at the time of their death.

Host and clinical factors associated with IMI in children with hematologic malignancies

Considering all cases with IMI compared with those who did not develop IMI, Hispanic ethnicity was associated with an almost two-fold risk of developing an IMI (OR: 1.94 [1.03-3.66], p=0.04). We also observed a $67 \%$ reduction in risk for cases diagnosed from 2016-2020 (OR: 0.33 [0.16-0.69], $\mathrm{p}=0.003$ ). Children with lymphoma were less likely to develop IMI $(\mathrm{p}=0.03)$ (Table 5 ).

\section{DISCUSSION}

To date, the majority of investigations of IMI in children have focused on infections resulting from a specific 
organism, such as invasive aspergillosis, or on very high-risk groups, such as HSCT recipients. Despite substantial evidence supporting the use of antifungal prophylaxis with mold activity in at-risk pediatric cancer populations, few studies have assessed the impact of appropriate prophylaxis on IMI incidence outside of a clinical trial, and none have detailed risk-adapted strategies for guidelines implementation and the subsequent impact on rates of IMI. In this 15-year single-institution study, the overall incidence of IMI in children treated for hematopoietic malignancy with chemotherapy alone was $4.6 \%$, ranging from a low of $1.7 \%$ for a diagnosis of lymphoma to a high of $6.4 \%$ for a diagnosis of AML. This rate approximates the frequency of IMI previously reported in U.S.- and European-based childhood cancer centers. ${ }^{10,11}$

Our results suggest a higher risk for IMI in children with Hispanic ethnicity. Factors contributing to the higher rates of IMI observed in Hispanics are likely multifactorial. A higher than expected incidence of infections with Histoplasma, Coccidioides, and Blastomycesspecies has been observed in persons of African, Native American, and Asian descent, ${ }^{12}$ suggesting a role for genetic predisposition. ${ }^{13}$ Further investigation to elucidate the genetic and environmental factors that underlie the excess IMI risk observed among Hispanic children is needed.

We also demonstrate clinical benefit to implementation of a standardized approach to antifungal prophylaxis in high risk childhood cancer populations, evidenced by risk reduction for proven/probable IMI after algorithm implementation. Our results validate and extend findings from a prior, smaller single-institution study that also reported a reduction in IMI incidence after applying risk-adapted IMI prophylaxis in children with leukemia. ${ }^{14}$ Antifungal prophylaxis is routinely prescribed to prevent IMI in children with AML, who are at high risk of infection due to prolonged periods of profound neutropenia induced by intensive chemotherapy. By broadening prophylaxis to include anti-mold coverage, we observed a reduction in the incidence of IMI in patients with AML treated at TCH by $60 \%$. Some adult studies have observed an increase in Mucor spp. infections with use of broadened, anti-mold prophylaxis. ${ }^{15,16}$ Although it is difficult to assess the effect of broadening prophylaxis in a sample of this size, we did observe an increase in RhizopusIMI postimplementation, one of the species in the Mucorales order. Of the proven IMI cases, Rhizopus or Mucor spp. were isolated in five out of 40 cases from 2006-2015 (13\%), vs. four out of 13 cases between 2016-2020 (31\%). Overall, concurrent to eliminating IMIs related to Aspergillus, we saw an increase in IMIs due toRhizopus , Fusarium, and Trichosporon ${ }^{17}$ that did not impact overall survival, but that did correspond with a higher rate of admission to the intensive care unit after 2016, and, for those who died, a higher rate of active fungal disease at time of death. Proven or probable IMI had a substantial impact on survival: only $27 \%$ of children with AML and $67 \%$ of children with ALL and concurrent IMI survived, compared with current average U.S. survival rates that approach $70 \%$ and $90 \%$, for AML and ALL respectively. ${ }^{18,19}$

The Children's Oncology Group recommends primary antifungal prophylaxis for children receiving HSCT as well as for patients with AML or myelodysplastic syndrome, with moderate evidence also suggesting benefit to primary prophylaxis for children with anticipated neutropenia greater than 7 days duration. ${ }^{20} \mathrm{As}$ noted above, a recent systematic review produced consensus guidelines for systemic antifungal prophylaxis in children with cancer and who are treated with HSCT that extends these recommendations to include moldactive antifungal prophylaxis for children treated for AML, and consideration of mold-active antifungal prophylaxis in children with newly diagnosed or relapsed ALL who are at high risk for fungal infection. ${ }^{8}$ Though our antifungal prophylaxis algorithm (Figure 1 ) was developed prior to the publication of these consensus guidelines, the algorithm largely adheres to the guideline principles. Specifically, according to our algorithm, antifungal prophylaxis with agents that have anti-mold activity is initiated for all patients diagnosed with AML, relapsed ALL or AML, and initiated during all phases of ALL therapy when inpatient admission through count recovery is recommended. Children with lymphoma are not prescribed antifungal prophylaxis. In addition, the TCH algorithm suggests appropriate anti-mold coverage to minimize potential interactions with chemotherapy agents routinely employed in subsets of ALL and AML, and considers variable absorption and pharmacokinetics of voriconazole and posaconazole in children. ${ }^{21}$ Exceptions to the published guidelines include our use of antifungal agents that are not mold-active (e.g. fluconazole) for specific moderate risk populations, such as during intensive treatment courses for children with Down syndrome associated ALL, infant ALL, and T cell ALL, as well as during periods of hyperglycemia for patients who develop 
steroid-induced diabetes during treatment. Importantly, the algorithm is a 'living' document, modified as treatment protocols are retired or initiated, as changes are made to the hospital formulary, or as new categories of risk are identified.

Limitations to our study include the anticipated constraints of a retrospective chart review, and the study restriction to a single institution. The relative rarity of this outcome precluded our ability to assess associations and outcomes for individual mold species. We also had insufficient data available to compare antimicrobial resistance patterns in isolated molds pre and post-algorithm implementation. Last, while our antifungal prophylaxis algorithm is evidence-based and largely aligned with current consensus guidelines, adaptations were made in consideration of prevalent fungal pathogens at our institution, additional populations perceived as high risk, and clinical practice preferences for intravenous vs. oral antimicrobials depending on inpatient or outpatient status. These adaptations should be considered when assessing the generalizability of our approach.

As noted by Lehrnbecher et al., the practical application of clinical guidelines requires an appreciation of local IFD epidemiology and the appropriate engagement and education of key stakeholders. ${ }^{8}$ Here, we describe an approach for the clinical application of evidence-based recommendations to provide antifungal prophylaxis in children with hematologic malignancies that is informed by host and treatment factors, local IMI epidemiology, and input from a multidisciplinary team. Further, we demonstrate a reduction in IMI incidence as clinical evidence of the efficacy of our approach. Ongoing efforts include continued prospective monitoring of fungal species isolated from at-risk populations to assess susceptibility patterns, outcomes, and the need for further algorithm modifications.

Acknowledgements: The study team would like to thank the patients and families who contributed data to this research. This work was supported by a St. Baldrick's Foundation Consortium Grant to Karen R. Rabin (Reducing Ethnic Disparities in Acute Leukemia), and by an American Society of Hematology Minority Medical Student Award to Ashley Ikwuezunma (mentors Michael E. Scheurer and Maria M. Gramatges).

Data Sharing: De-identified data that support the findings of this study are available on request from the corresponding author. The data are not publicly available due to privacy or ethical restrictions.

\section{References}

1. Groll AH, Castagnola E, Cesaro S, et al. Fourth European Conference on Infections in Leukaemia (ECIL4): guidelines for diagnosis, prevention, and treatment of invasive fungal diseases in paediatric patients with cancer or allogeneic haemopoietic stem-cell transplantation. Lancet Oncol. 2014;15(8):e327-340.

2. Fisher BT, Robinson PD, Lehrnbecher T, et al. Risk Factors for Invasive Fungal Disease in Pediatric Cancer and Hematopoietic Stem Cell Transplantation: A Systematic Review.J Pediatric Infect Dis Soc. 2018;7(3):191-198.

3. Lehrnbecher T, Ethier MC, Zaoutis T, et al. International variations in infection supportive care practices for paediatric patients with acute myeloid leukaemia. Br J Haematol. 2009;147(1):125-128.

4. Ninane J. A multicentre study of fluconazole versus oral polyenes in the prevention of fungal infection in children with hematological or oncological malignancies. Multicentre Study Group. Eur J Clin Microbiol Infect Dis. 1994;13(4):330-337.

5. Caselli D, Arico M, Michelone G, Cavanna C, Nespoli L, Burgio GR. Antifungal chemoprophylaxis in cancer children: a prospective randomized controlled study.Microbiologica. 1990;13(4):347-351.

6. Fisher BT, Zaoutis T, Dvorak CC, et al. Effect of Caspofungin vs Fluconazole Prophylaxis on Invasive Fungal Disease Among Children and Young Adults With Acute Myeloid Leukemia: A Randomized Clinical Trial. Jama. 2019;322(17):1673-1681.

7. Sawada A, Sakata N, Higuchi B, et al. [Comparison of micafungin and fosfluconazole as prophylaxis for invasive fungal infection during neutropenia in children undergoing chemotherapy and hematopoietic stem 
cell transplantation].Rinsho Ketsueki. 2009;50(12):1692-1699.

8. Lehrnbecher T, Fisher BT, Phillips B, et al. Clinical Practice Guideline for Systemic Antifungal Prophylaxis in Pediatric Patients With Cancer and Hematopoietic Stem-Cell Transplantation Recipients. J Clin Oncol.2020;38(27):3205-3216.

9. De Pauw B, Walsh TJ, Donnelly JP, et al. Revised definitions of invasive fungal disease from the European Organization for Research and Treatment of Cancer/Invasive Fungal Infections Cooperative Group and the National Institute of Allergy and Infectious Diseases Mycoses Study Group (EORTC/MSG) Consensus Group.Clinical infectious diseases : an official publication of the Infectious Diseases Society of America. 2008;46(12):1813-1821.

10. Lehrnbecher T, Schoning S, Poyer F, et al. Incidence and Outcome of Invasive Fungal Diseases in Children With Hematological Malignancies and/or Allogeneic Hematopoietic Stem Cell Transplantation: Results of a Prospective Multicenter Study.Front Microbiol. 2019;10:681.

11. Cesaro S, Tridello G, Castagnola E, et al. Retrospective study on the incidence and outcome of proven and probable invasive fungal infections in high-risk pediatric onco-hematological patients. Eur J Haematol. 2017;99(3):240-248.

12. Bongomin F, Gago S, Oladele RO, Denning DW. Global and Multi-National Prevalence of Fungal Diseases-Estimate Precision. J Fungi (Basel). 2017;3(4).

13. Merkhofer RM, Klein BS. Advances in Understanding Human Genetic Variations That Influence Innate Immunity to Fungi. Front Cell Infect Microbiol. 2020;10:69.

14. Meryk A, Kropshofer G, Hutter J, et al. Benefits of risk-adapted and mould-specific antifungal prophylaxis in childhood leukaemia. Br J Haematol.2020;191(5):816-824.

15. Ananda-Rajah MR, Grigg A, Slavin MA. Breakthrough disseminated Scedosporium prolificans infection in a patient with relapsed leukaemia on prolonged voriconazole followed by posaconazole prophylaxis. Mycopathologia. 2008;166(2):83-86.

16. Georgiadou SP, Pongas G, Fitzgerald NE, et al. Invasive Mold Infections in Pediatric Cancer Patients Reflect Heterogeneity in Etiology, Presentation, and Outcome: A 10-Year, Single-Institution, Retrospective Study. Journal of the Pediatric Infectious Diseases Society. 2012;1(2):125-135.

17. Foster CE, Edwards MS, Brackett J, Schady DA, Healy CM, Baker CJ. Trichosporonosis in Pediatric Patients With a Hematologic Disorder. J Pediatric Infect Dis Soc.2018;7(3):199-204.

18. Elgarten CW, Aplenc R. Pediatric acute myeloid leukemia: updates on biology, risk stratification, and therapy. Current opinion in pediatrics. 2020;32(1):57-66.

19. Hunger SP, Mullighan CG. Acute Lymphoblastic Leukemia in Children. $N$ Engl $J$ Med.2015;373(16):1541-1552.

20. Science M, Robinson PD, MacDonald T, Rassekh SR, Dupuis LL, Sung L. Guideline for primary antifungal prophylaxis for pediatric patients with cancer or hematopoietic stem cell transplant recipients. Pediatric blood \& cancer.2014;61(3):393-400.

21. Dvorak CC, Fisher BT, Sung L, et al. Antifungal prophylaxis in pediatric hematology/oncology: new choices \& new data. Pediatric blood $\mathscr{E}$ cancer. 2012;59(1):21-26.

\section{FIGURE LEGEND}

Figure 1 : Texas Children's Hospital evidence-based, risk-adapted algorithm for antifungal prophylaxis in children with hematologic malignancies. 


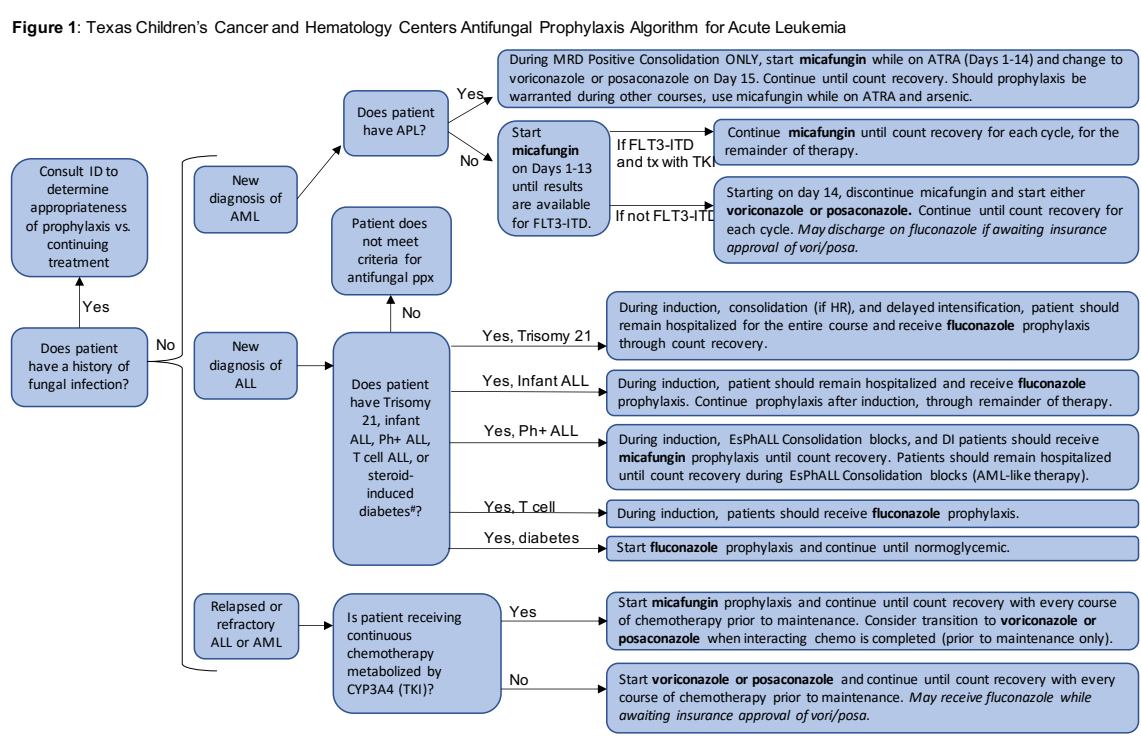

ALL: Acute lymphoblastic leukemia, AML: Acute myeloid leukemia, APL: Acute promyelocytic leukemia, FLT3-ITD: FLT3 internal tandem duplication "Steroid-induced diabetes $=2 \mathrm{hr}$ post-prandial glucose $\geq 200$ or fasting glucose $\geq 126$ (without dextrose in IVF)

\section{Hosted file}

Table 1_05_03_21.pdf available at https://authorea.com/users/412093/articles/520891-anevidence-based-risk-adapted-algorithm-for-anti-fungal-prophylaxis-reduces-risk-forinvasive-mold-infections-in-children-with-hematologic-malignancies

\section{Hosted file}

Table 2_05_03_21.pdf available at https://authorea.com/users/412093/articles/520891-anevidence-based-risk-adapted-algorithm-for-anti-fungal-prophylaxis-reduces-risk-forinvasive-mold-infections-in-children-with-hematologic-malignancies

\section{Hosted file}

Table 3_05_03_21.pdf available at https://authorea.com/users/412093/articles/520891-anevidence-based-risk-adapted-algorithm-for-anti-fungal-prophylaxis-reduces-risk-forinvasive-mold-infections-in-children-with-hematologic-malignancies

\section{Hosted file}

Table 4_05_03_21.pdf available at https://authorea.com/users/412093/articles/520891-anevidence-based-risk-adapted-algorithm-for-anti-fungal-prophylaxis-reduces-risk-forinvasive-mold-infections-in-children-with-hematologic-malignancies

\section{Hosted file}

Table 5_05_03_21.pdf available at https://authorea.com/users/412093/articles/520891-anevidence-based-risk-adapted-algorithm-for-anti-fungal-prophylaxis-reduces-risk-forinvasive-mold-infections-in-children-with-hematologic-malignancies 